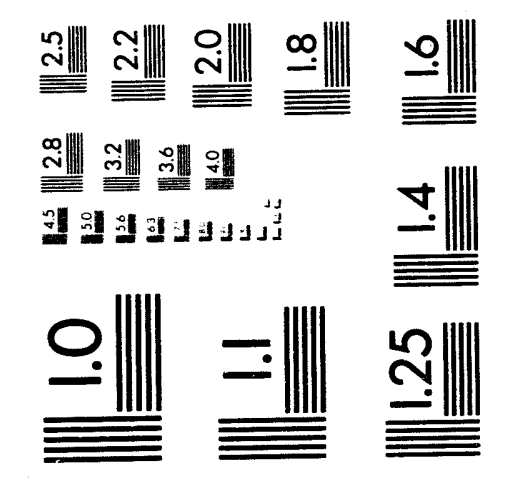



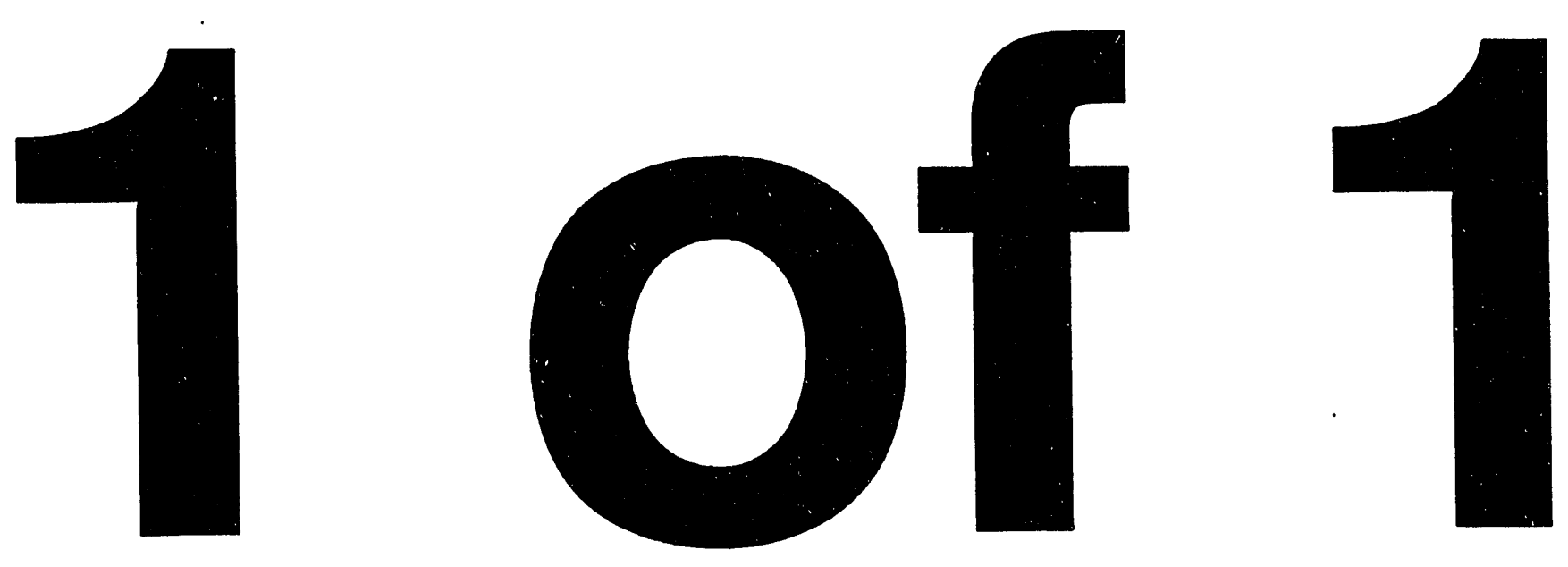
Paper to be presented at the IEEE Nuclear and Space

BNL-60072

Radiation Effects Conference,

Tucson, Arizona, July 18-22, 1994

To be published in the IEEE Transaction on Nuclear Science

\title{
Determination of the Fermi Level Position for Neutron Irradiated High Resistivity Silicon Detectors and Materials Using the Transient Charge Technique(TChT)*
}

\author{
V. Eremin ${ }^{+}$and Z. Li \\ Brookhaven National Laboratory \\ Upton, NY 11973, USA
}

+: Permanent address: A.F. Ioffe Physico-Technical Institute of Academy of Sciences of Russia

St. Petersburg, Russia

\section{DISCLAIMER}

\begin{abstract}
This report was prepared as an account of work sponsored by an agency of the United States Government. Neither the United States Government nor any agency thereof, nor any of their employees, makes any warranty, express or implied, or assumes any legal liability or responsibility for the accuracy, completeness, or usefulness of any information, apparatus, product, or process disclosed, or represents that its use would not infringe privately owned rights. Reference herein to any specific commercial product, process, or service by trade name, trademark, manufacturer, or otherwise does not necessarily constitute or imply its endorsement, recommendation, or favoring by the United States Government or any agency thereof. The views and opinions of authors expressed herein do not necessarily state or reflect those of the United States Government or any agency thereof.
\end{abstract}

February, 1994

*This research was supported in part by the U. S. Department of Energy: Contract No. DE-AC02-76CH00016, and by the National Research Council: Grant \# LI-CAST93 


\section{Determination of the Fermi Level Position for Neutron Irradiated High Resistivity Silicon Detectors and Materials Using the Transient Charge Technique(TChT)*}

\author{
V. Eremin ${ }^{+}$and $\mathrm{Z} . \mathrm{Li}$ \\ Brookhaven National Laboratory \\ Upton, NY 11973, USA
}

\section{t: Permanent address: A.F. Ioffe Physico-Technical Institute of Academy of Sciences of Russia \\ St. Petersburg, Russia}

\section{Abstract}

The transient charge technique (TChT) has been used in this work to study the electrical properties in both space charge region (SCR) and electrical neutral bulk (ENB) of neutron irradiated high resistivity $(4-6 \mathrm{k} \Omega-\mathrm{cm})$ silicon particle detectors. Detectors irradiated to various neutron fluences were measured by TChT at various biases and temperatures below the room temperature. The Fermi level, obtained from the Arrhenius plot of the time constant of the slow component of the charge shape, has been found to stabilize around $E_{c}-0.47$ to $0.50 \mathrm{eV}$ at high fluences $(\Phi$ $n>10^{13} \mathrm{n} / \mathrm{cm}^{2}$ ). The resistivity of the ENB has been found greater than $300 \mathrm{k} \Omega-\mathrm{cm}$ at high fluences.

\section{Summary}

Recently, much of the interest on the neutron radiation effects on high resistivity $p^{+/ n}$ junction detectors have been concentrated on the high fluence ( $\Phi_{n}>10^{13} \mathrm{n} / \mathrm{cm}^{2}$ ) neutron induced changes of detector and material electrical properties, in the wake of the anticipated high radiation environment $\left(10^{14} \mathrm{n} / \mathrm{cm}^{2}\right.$ in ten years) in the newly proposed Large Hadron Collider. The effective doping concentration (sometime also called : effective impurity concentration) $\mathrm{N}_{\text {eff }}$ in the space charge region (SCR), which determines the detector full depletion voltage $V_{d}$, as a function of neutron fluence has been mainly studied by the capacitance-voltage (C-V) technique $[1-4]$, although there are some recent attempts using the transient current techniques (TCT), such as alpha[5] and laser [6] induced current shapes, to obtain $\mathrm{V}_{d}$ and $\mathrm{N}_{\text {eff. The }}$ $\mathrm{C}-\mathrm{V}$ technique becomes less reliable at high neutron fluences due to high leakage current and frequency dependence[2]. The material properties, or the electrical neutral bulk (ENB) properties, have been studied by entirely different techniques such as the four-point probe approach [7], the resistor structure approach $[8]$, and the Hall effect approach $[9-10]$. All these approaches, however, are highly limited by the surface conditions and by the contact problems (ohmic contacts required). In this paper, we will present the data of detector SCR properties such as $\mathrm{N}_{\text {eff, }}$ and ENB properties such as the position of the Fermi level , resistivity, and free carrier concentrations (n and/or p) as a function of neutron fluence using the transient charge technique (TChT).

The laser TCT/TChT set-up in BNL, designed in PTI and improved in BNL under the current PTIBNL collaboration, is consist of a GaAs laser diode ( $\lambda$ $\sim 0.82 \mu \mathrm{m}$ ), a nanosecond pulse generator and a current amplifier supplying laser pulse, an oscilloscope, a temperature controller, and a $\mathrm{L}-\mathrm{N}_{2}$ dewar for low temperature measurements.

An example of the laser induced current shape is shown in Fig. 1 for a non-irradiated detector. The detector was biased in a partially depleted mode and the laser illumination was on the $p^{+}$side for which the current shape is mainly electron induced current. For a non-irradiated detector, the electron current shape follows the exponential decay $e^{-t / \tau}$ and the time constant can be written as $[6,11]$ :

$\tau=\frac{\varepsilon \varepsilon_{0}}{e \mu N_{\text {eff }}} \cdot \frac{d}{w}$

where $\mathbf{d}$ is the detector thickness, $w$ the depletion width:

$w=\sqrt{\frac{2 \varepsilon \varepsilon_{0} V}{e N_{e f f}}}$

where $\mathrm{V}$ is the detector bias. The time constant for a non-irradiated detector is therefore dependent on both $\mathrm{V}$ and $\mathrm{N}_{\mathrm{eff}}$ :

$\tau=\frac{d}{\mu} \cdot \sqrt{\frac{\varepsilon \varepsilon_{0}}{2 e N_{e f f}}} \cdot \frac{1}{\sqrt{V}}$

Fig. 2 shows $1 / \tau^{2}$ for a non-irradiated detector as a function of the detector bias $V$. A clear linear dependence gives a $\mathrm{N}_{\text {eff }}$ of about $7.3 \times 10^{11} / \mathrm{cm}^{3}$, very 
close to the value of $7.0 \times 10^{11} / \mathrm{cm}^{3}$ obtained by the C-V method.

The charge shapes for an irradiated $\left(\Phi_{n}>10^{13}\right.$ $\mathrm{n} / \mathrm{cm}^{2}$ ) detector at various bias at room temperature (RT) are shown in Fig. 3. It is clear that there are two components in the charge shape: a fast one that is associatei with the drift current in the SCR (with a time constant $\tau_{1}$ ) and a slow one for which the time constant $\tau_{2}$ is related to the Maxwell relaxation time constant $\tau_{\mathrm{m}}$ as the following $[6,11]$ :

$\tau_{2}=\tau_{m} \cdot \frac{d}{w}$

where

$\tau_{m}=\frac{\varepsilon \varepsilon_{0}}{e \mu n(\operatorname{or} p)}$

We note here that for a given bias, $\tau_{2}$ depends on the temperature $\mathrm{T}$ through $\mathrm{n}$ or $\mathrm{p}$ :

$$
\tau_{2} \propto\left\{\begin{array}{ll}
e^{-\frac{E_{c}-E_{f}}{k T}} & (\mathrm{n}-\text { type }) \\
e^{-\frac{E_{f}-E_{v}}{k T}} & (\mathrm{p}-\text { type })
\end{array} \equiv e^{-\frac{E_{r}}{k T}}\right.
$$

It follows from Fig. 3 that the amplitude of the fast component (the drift component) $A_{d r}$ of the charge shape increases with the bias $V$ before the full depletion, and saturates at the full depletion. In fact, $A_{d r}$ is proportional to $V^{1 / 2}$ when $V<V_{d}$ :

$$
A_{d r}= \begin{cases}C \sqrt{V} & \left(V<V_{d}\right) \\ A_{d r}^{\max } & \left(V>V_{d}\right)\end{cases}
$$

Fig. 4 shows the measured $A_{d r}$ for the same detector shown in Fig. 3 as a function of $v^{1 / 2}$. The extrapolation of the linear part into the saturation gives the full depletion value $V_{d}=110$ volts, which in turn gives the value of $\mathrm{N}_{\mathrm{eff}}=1.60 \times 10^{12} / \mathrm{cm}^{2}$ using eq.(2) with $w=d$.

The charge shapes for the same detector shown in Fig.3 and 4, operated at a given bias at various temperatures are shown in Fig. 5. It is clear that the time constant of the slow component $\tau_{2}$ decreases drastically with the temperature. Arrhenius plots of $\tau_{2}$ for detectors irradiated to $3.2 \times 10^{13} \mathrm{n} / \mathrm{cm}^{2}$ and
$5.89 \times 10^{14} \mathrm{n} / \mathrm{cm}^{2}$ are shown in Fig. 6, which gives the Fermi level positions of $E_{F}=0.483 \mathrm{eV}$ and $E_{F}=0.498$ eV, respectively. As shown in Fig. 7, the Fermi level is stabilized near the middle gap at all neutron fluences under study $\left(\Phi_{n}>3.0 \times 10^{13} \mathrm{n} / \mathrm{cm}^{2}\right)$, similar to the findings in ref.[9]. The ENB resistivity was found also increasing with neutron fluence and tends to saturate at a value higher than the intrinsic value $(>300 \mathrm{k} \Omega-\mathrm{cm})$, in good agreement with ref. [6-8].

*This research was supported in part by the U.S. Department of Energy:

Contract No. DE-AC02-76CH00016, and by the National Research Council, Grant * LI-CAST93

\section{ACKNOWLEDGMENTS}

The authors would like to thank H.W. Kraner, T. Tsang, and S. Rescia of BNL for helpful discussions and comments to the improvement of the TCT/TChT system.

\section{REFERENCES}

1 G. Lindström, M. Benkert, E. Fretwurst, T. Schulz and R. Wunstorf, Proc. of the First International Conf. on Calorimetry in High Energy Physics, D.F. Anderson, et al., Des, (Word Scientific Publishing Co., Singapore, (1991) 467.

2 Z. Li, W. Chen, and H.W. Kraner, Nucl. Instr. and Meth. A308 (1991) 585.

3 R. Wunstorf, M. Benkert, N. Claussen, N. Croitoru, E. Fretwurst, G.Lindstrom, and T. Schulz, Results on Radiation Hardness of Silicon Detectors up to Neutron Fluence of $10^{15} \mathrm{n} / \mathrm{cm}^{2}$, Nucl. Instr. Meths., A315, 149 (1992)

4. K. Gill, G. Hall, S. Roe, S. Sotthibandhu, R. Wheadon, P. Giubellino, and L. Ramello, Radiation Damage by Neytrons and Photons to Silicon Detectors, Nucl. Instr. Meths., A322, 177 (1992).

5 H.W. Kraner, Z. Li, and E. Fretwurst, The use of the Signal Current Fulse Shape to Study the Internal Electrical Field Profile and Trapping effects in Neutron Damaged Silicon Detectors, Presented at the Sixth European Symposium on Semiconductor Detectors, Milano, Italy, Feb. 24 26, 1992, Nucl. Instr. and Meth., A326 (1993) 350-356.

6 Z. Li, V. Eremin, N. Strokan, and E. Verbitskaya, Investigation of the Type Inversion 
Phnomena: Resistivity and Carrier Mobility in the Space Charge Region and Electrical Neutral Bulk in Neutron Irradiated Silicon $p^{+}-n$ Junction Detectors, Presedted at the IEEE Nucl. Sci. Symp, Orlando, FL, Oct. 25-31, 1992, Trans. Nucl. Sci. Vol. 40, No.4, 367(1993)

7 I. Tsvybak, B. Bugg, J. Walter, and J.A. Harvey, Fast Neutron-Induced Changes in Net Impurity Concentration of High Resistivity Silicon, Trans. Nucl. Sci., Vol. 39, No.6, 1720(1992).

8. Z. Li, Resitivity Measurements on Neutron Irradiated Detector Grade Silicon Materials, Presented at the 1st International Conference on Large Scale Application and Radiation Hardness of Semiconductor Detectors, Florence, Italy, July 7 -

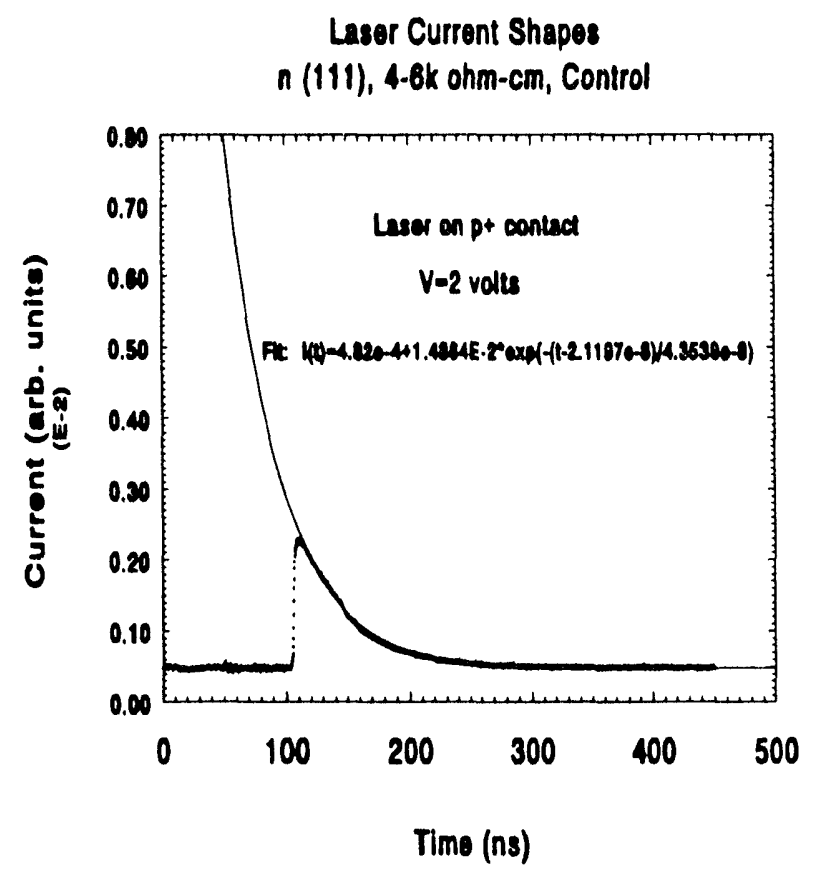

Fig. 1 Laser induced current shape for a nonirradiated detector $\left(\Phi_{\mathrm{n}}=0.0 \mathrm{n} / \mathrm{cm}^{2}\right)$. The laser illumination was on the $\mathrm{p}^{+}$side of the detector.
9, 1993, to be published in Nuov Cimento (in press).

9 P.F. Lugakov, T.A. Lukashevich, and V.V Shussha, Phys. Sta. Sol., A, Vol. 74, 445(1982).

10 U. Biggeri, E. Borchi, M. Bruzzi, S. Lazanu, Z. $\mathrm{Li}$, and $\mathrm{R}$. Beuttenmuller, Type Invertion Measurements on Irradiated Silicon by Means of Hall Effect, Presented at the 1st International Conference on Large Scale Application and Radiation Hardness of Semiconductor Detectors, Florence, Italy, July 7-9, 1993, to be published in Nuov Cimento (in press).

11 V. Eremin, N. Strokan, and N. tisnek, Sov. Phys. Semicond., Vol. 8, 1157(1974)

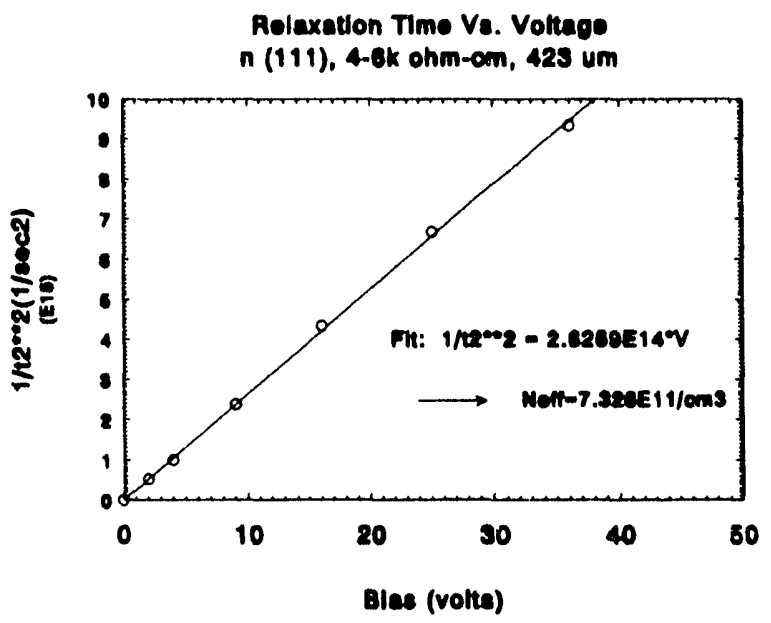

Fig. 2 The time constant $\tau$ obtained from fitting the exponential decay of the current shape of a nonirradiated detector as a function of the detector bias.

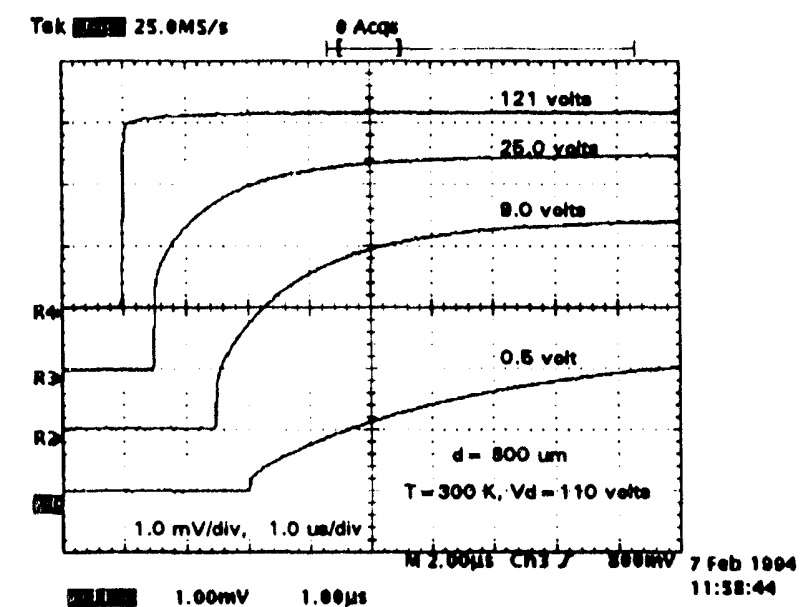

Fig. 3 Charge shapes for an irradiated detector $\left(\Phi_{n}=3.29 \times 10^{13} \mathrm{n} / \mathrm{cm}^{2}\right)$ at RT with different operating biases. Laser illumination was on the $n^{+}$ side of the detector. 
Amplitude of the Dritf Charge

n (111), 4-6k ohm-cm, 300 um

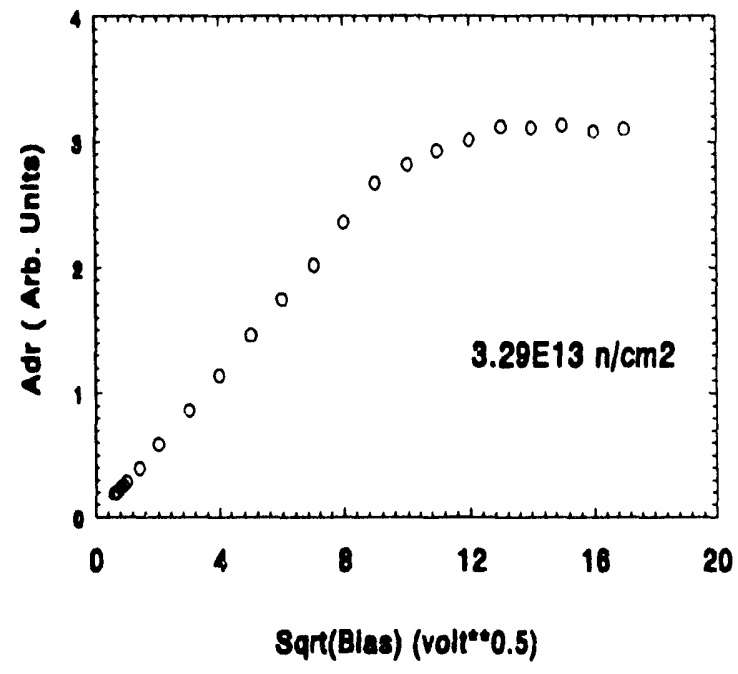

Fig. 4 Amplitude of the fast component $\left(A_{d r}\right)$ in the charge shape as a function of the square root of the bias for an irradiated detector $\left(\Phi_{n}=3.29 \times 10^{13}\right.$ $\left.\mathrm{n} / \mathrm{cm}^{2}\right) . A_{d r}$ saturates at the full depletion.

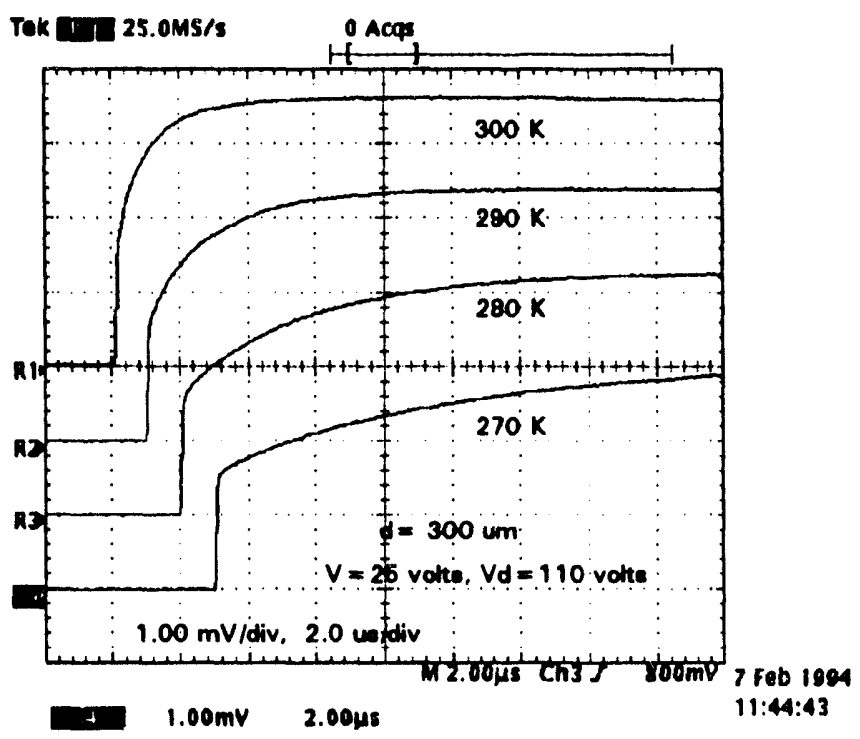

Fig. 5 Charge shapes for an irradiated detector $\left(\Phi_{\mathrm{n}}=3.29 \times 10^{13} \mathrm{n} / \mathrm{cm}^{2}\right)$ operated at a given bias at various temperatures. Laser illumination was on the $\mathrm{n}^{+}$side of the detector. $n(111)$ 4-6k, 300um

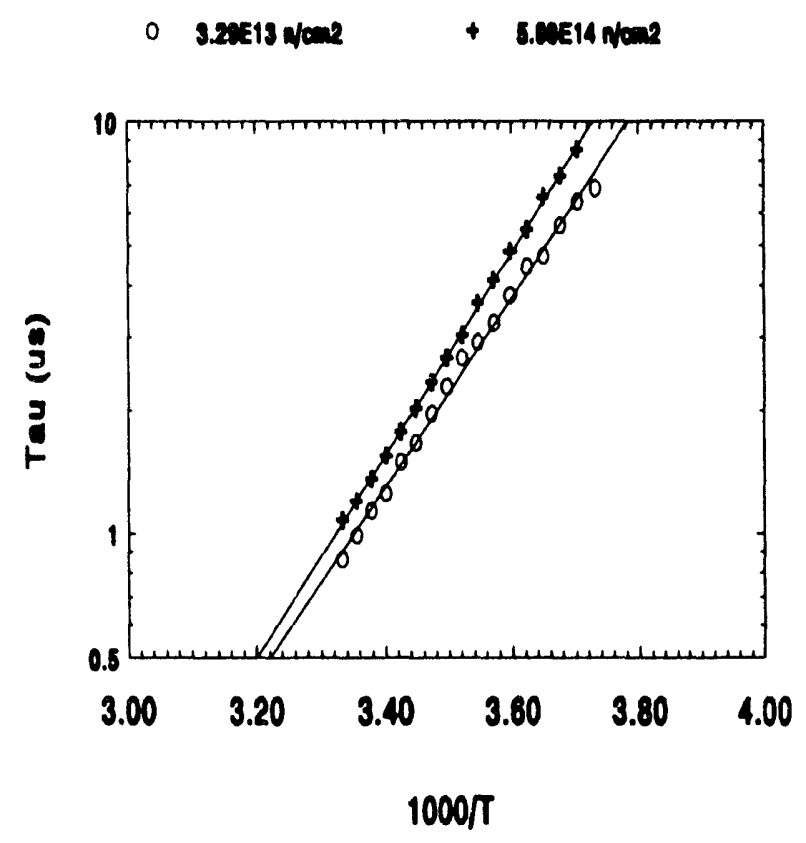

Fig. 6 Arrehenius plots of $\tau_{2}$ for two detectors irradiated to different fluences.

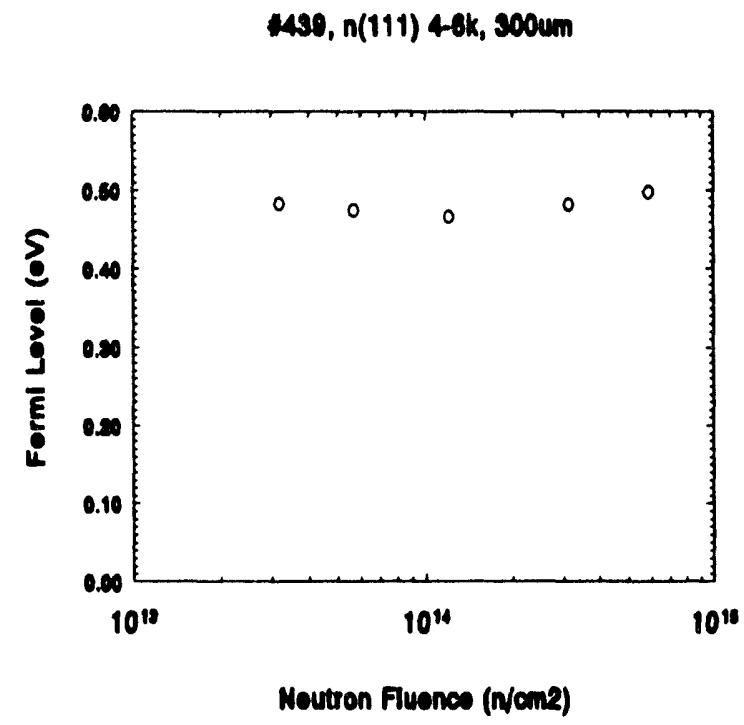

Fig. 7 Dependence of the Fermi level position on the neutron fluence. 

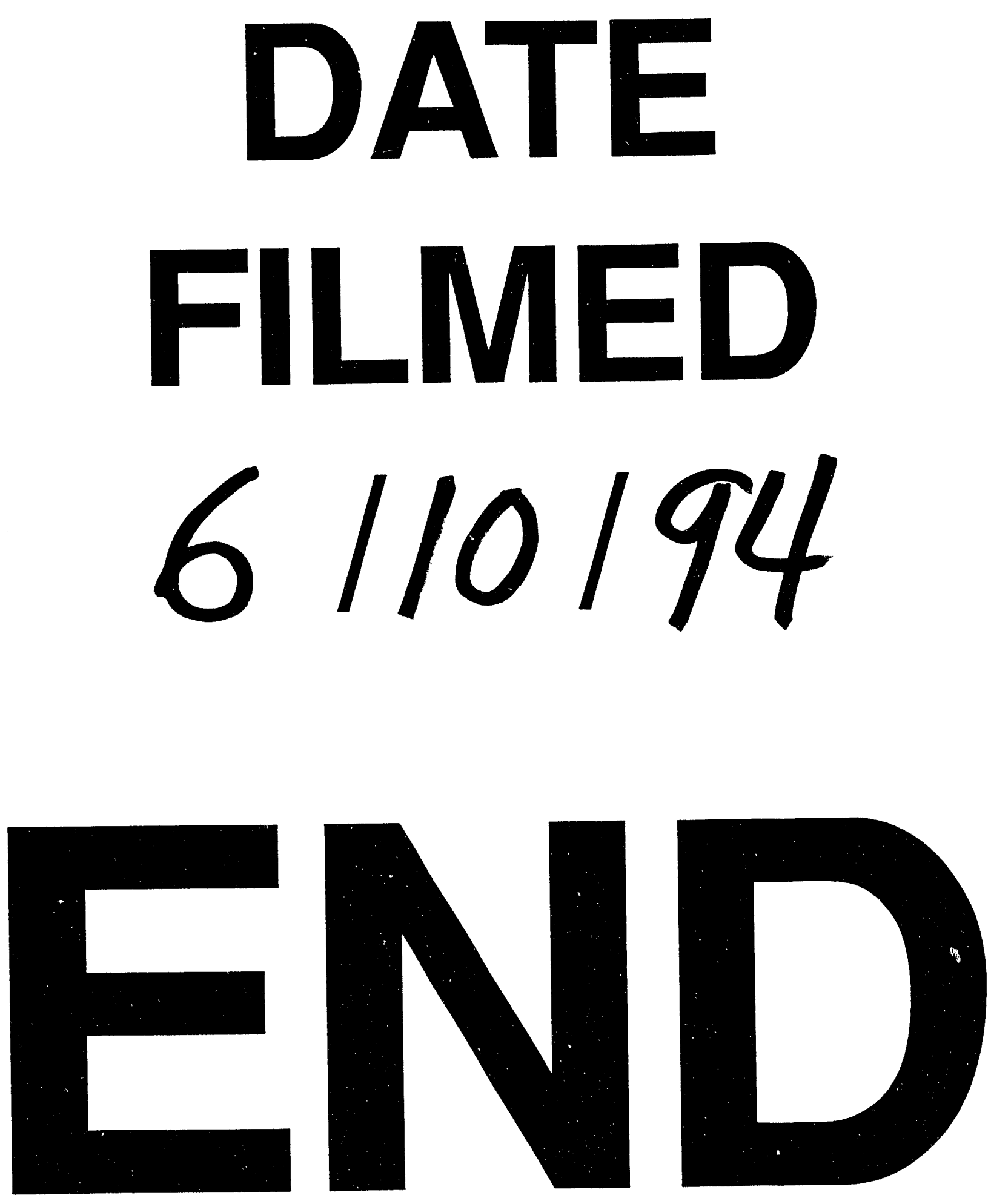
